\title{
Smart Technology for Gesture Recognition Using Accelerometer
}

\author{
Priyanka Rajmane ${ }^{1}$, Nimish Bendre ${ }^{2}$, Maitreyie Chavan $^{3}$, Sriraksha Deshpande ${ }^{4}$, Akshay Dhende \\ ${ }^{1}$ Assistant Professor, Department of Computer Engineering, MES College of Engineering \\ 2,3,4,5 MES College of Engineering, Pune University, Pune, India
}

\begin{abstract}
Patients in hospitals often have to rely on others for assistance. Remote controlled devices can help such people. Our Project presents the implementation of an accelerometer enabled mobile device which can be used by patients to assist them with room maintenance, call for food and medicines, etc. with a simple mobile gesture. The main objective is to develop a low cost device which can be developed/implemented with off the shelf components. The novelty of this device is its low cost. The device can be used and implemented by patients easily. For hand gesture recognition, a HMM (Hidden Markov Model) has been used to model the sequential constraints on the gestures, which increases the recognition accuracy.
\end{abstract}

Keywords: Gesture recognition, Interactive controller, MEMS accelerometer, Assisted living, Hidden Markov model (HMM), Human Robot Interaction (HRI)

\section{Introduction}

This paper presents the implementation of an accelerometer enabled mobile device which can be used by patients to assist them with room maintenance, call for food and medicines, etc. with a simple mobile gesture. The main objective is to develop a low cost device which can be developed/implemented with off the shelf components. The system is being developed keeping in mind the patients in hospitals, hence the area of the project will be hospitals. It will improve the communication between patients and their doctors/nurses and also help the hospitals improve their services. Since the physically challenged patients have to rely on others for help, the motivation was developing a device which helps them to get the required without depending on anybody.

Bed-ridden patients will use their mobile devices and make one of the following gestures:

Left tilt: Nurse assistance

Right tilt: Demand for food and medicines

Up and Down tilt: Room maintenance

Vigorous Shake: Critical problem

Users will be making one of the above mentioned gestures. No other gestures will be recognized by the system. Users will not be able to use the mobile device for any other purpose except for the devices programmed features.

\section{Goals and objectives}

- Low cost device to help patients get assistance.

- Prevents situations from reaching a critical point

- Keeps the nurses and doctors up to date

- A priority based algorithm for prioritizing the tasks efficiently

- Dedicated server which should keep a track on the management of notifications and actions taken

- On receiving a critical alert, it has to be made sure that some doctor has attended the patient and his/her condition is stabilized

\section{Requirements}

\begin{tabular}{|c|c|c|c|}
\hline Sr. No. & Parameter & Minimum Requirement & Remarks \\
\hline 1 & CPU Speed & $2.4 \mathrm{GHz}$ & Required \\
\hline 2 & HDD & $80 \mathrm{~GB}$ & Required \\
\hline 3 & RAM & $512 \mathrm{MB}$ & Required \\
\hline 4 & PC's & 2 & Required \\
\hline 5 & Android Device & 2 & Required \\
\hline
\end{tabular}

\subsection{Software Requirements:}

- Operating System: Linux- Ubuntu / Fedora, Windows, Android

- Web Services: ReST/SOAP

- IDE: Eclipse NetBeans

- Programming Language Java $\mathrm{C}++$

\subsubsection{Web Services:}

- REST is a web service. Everything is a resource in this web service. RESTful web services are scalable, having light weight and easy to maintain. They are commonly used to create APIs for web based applications.

- SOAP Simple Object Access Protocol is used as a messaging protocol. It allows programs running on separate operating systems to communicate using XML and HTTP.

\section{Hidden Markov Model}

This system consists of data collection from sensors, segmentation and recognition. After receiving acceleration

\section{Volume 4 Issue 12, December 2015}




\section{International Journal of Science and Research (IJSR) \\ ISSN (Online): 2319-7064}

Index Copernicus Value (2013): 6.14 | Impact Factor (2014): 5.611

data from the device, HMM algorithm is applied to determine the start and end points of every input gesture automatically. The sequence of gesture is extracted as a gesture code. The gesture code is compared with the stored gesture patterns to determine the most likely gesture. For hand gesture recognition, an HHMM has been used to model the sequential constraints on the gestures, which increases the accuracy of recognizing the gesture. After reviewing the different techniques for recognition of hand gesture, recognition of hand gesture through Accelerometer is the best technique for this system.

A HMM is a set of finite states linked by transitions. Each state is categorized by two sets of probabilities: transition, discrete or continuous output probability which defines the condition probability of resulting each output symbol from an alphabet or continuous vector. A HMM can be defined by:

$0\{\mathrm{~S}\}$ - set of states, including a final state SF and an initial state SI

$0\{\mathrm{~A}\}$ - transition probability matrix,

$\mathrm{A}=\{\mathrm{axy}\}$, where axy is the transition probability of taking transition from state $\mathrm{z}$ to state $\mathrm{j}$,

$\mathrm{B}=$ The output probability matrix,

$\mathrm{B}=\{\mathrm{bj}(\mathrm{O})$,$\} for discrete HMM,$

$\mathrm{B}=\{\mathrm{bj}(\mathrm{x})\}$ for continuous HMM, where $\mathrm{O}$ stands for a discrete observation symbol, and $\mathrm{x}$ stands for continuous observations of $\mathrm{k}$-dimensional random vectors

\section{Basic Working}

\section{- USER (PATIENT).}

The patients can use the following gestures:

Right Tilt: Food and Medicines.

Left Tilt: Nurse Assistance.

Up and Down: Room maintenance.

Vigorous Shake: Critical state.

Once the user makes a particular gesture the value of the gesture will be generated and the database will be checked to see which doctor/nurse is assigned to that patient. No indepth knowledge of the system is required by the user.

\section{- Receivers}

There will be three receivers who can get the notification. The nurse will get the notification when the right, left tilt and vigorous shake is made. The doctor will get the notification when the vigorous shake is made. Upon the up and down movement a notification will be sent on that floor's LCD monitor that will highlight the patients room number in red which will inform the maids about the room that is asking for room service.

- Methodology

A simple working can be shown in the diagram below:

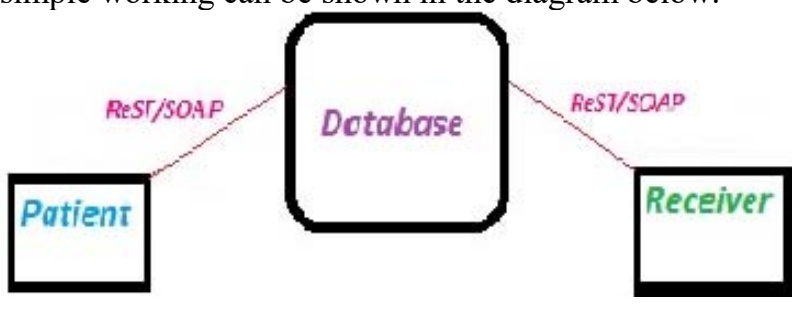

\section{Conclusion}

After a thorough reading of $\mathbf{1}$ - $\mathbf{5}$ we have reached to a conclusion that gesture recognition via accelerometer is the most effective method both technically and economically.

We use the Hidden Markov Model for the recognition module for its high accuracy and efficiency. The Device will be used for the intended purpose only and may have added features later on.

The device will have an easy interface for the users and will be monitored by a central server. The server will also foresee the assignment of the appropriate staff for the aroused situation. This system will help the all patients but will mainly aim to assist the elderly and bed ridden patients who cannot physically call for help in the time of need.

\section{Acknowledgement.}

We are deeply indebted to our guide Prof. Priyanka Rajmane whose guidance, stimulating suggestions and encouragement helping us in all the time of our paper.

\section{References}

[1] Zhiyuan Lu, Xiang Chen, Member, IEEE, Qiang Li. Xu Zhang, Member, IEEE and Ping Zhou, Member, IEEE, "A Hand Gesture Recognition Framework and Wearable Gesture-Based Interaction Prototype for Mobile Device" in IEEE transactions on humanmachine systems,vol.44,no.2,April 2014.J. Breckling, Ed., The Analysis of Directional Time Series: Applications to Wind Speed and Direction, ser. Lecture Notes in Statistics. Berlin, Germany: Springer, 1989, vol. 61.

[2] R. Xu, S. Zhou and W.J. Li., "MEMS accelerometer based nonspecific user hand gesture recognition", IEEE Sensors J., vol. 12, no. 5, pp. 1166-1173,May 2012.

[3] Xu Zhang, Xiang Chen, Associate Member, IEEE, Yun Li, Vuokko Lantz, Kongqiao Wang and Jihai Yang, “A Framework for Hand Gesture Recognition Based on Accelerometer and EMG Sensor", IEEE transactions on systems, man and cybernetics - part a: systems and humans, vol. 41, no. 6,November 2011.

[4] C. Zhu and W. Sheng, "Wearable sensor based hand gesture and daily activity recognition for robot- assisted living", IEEE Trans. Syst., Man, Cybern. A, Syst. Humans, vol. 41, no. 3, pp. 569-573, May 2011.

[5] The IEEE website. [Online]. Available: http://www.ieee.org/ 